\title{
Cuatro años de experiencia en tratamiento médico del embarazo ectópico en el departamento de Paysandú
}

Four-year experience in medical treatment of ectopic pregnancy in the department of Paysandú

Quatro anos de experiência no tratamento de gravidez ectópica no departamento de Paysandú

\section{Dres. Vanessa Guzzoํ, Sebastián Ben², Natalia Sica ${ }^{3}$}

\section{Resumen}

Introducción: en los últimos años se viene presentando un fenómeno de disminución en las defunciones vinculadas al embarazo ectópico gracias a los avances en el diagnóstico precoz, lo que a su vez abrió las puertas al tratamiento médico con metotrexate en pacientes seleccionadas. El objetivo del presente trabajo es reportar la experiencia del tratamiento con metotrexate en el departamento de Paysandú y determinar secundariamente la satisfacción de las usuarias frente a éste y su fertilidad posterior.

Método: se presenta un estudio retrospectivo, observacional, de los embarazos ectópicos tubarios tratados con metotrexate en Paysandú, durante el período del $1^{\circ}$ de enero de 2014 al 31 de diciembre de 2017, comprendiendo cuatro años. Se consideró fracaso del tratamiento médico cuando fue necesario tratamiento quirúrgico y se definió como fertilidad futura al tiempo que transcurrió hasta lograr una gestación intrauterina espontánea.

Resultados: se registraron 67 embarazos ectópicos en cuatro años, 13 recibieron metotrexate intramuscular con un porcentaje de éxito de $69,2 \%$. Se determinó una fertilidad posterior de $25 \%$.

Conclusiones: el uso de metotrexate en el embarazo ectópico se practica en Paysandú desde hace casi una década con buenos resultados y una buena aceptación por parte de las usuarias.

Palabras clave: Embarazo ectópico

Metotrexato

Fertilidad

Key words: $\quad$ Ectopic pregnancy

Methotrexate

Fertility

\footnotetext{
1. Asistente de Clínica Ginecotológica A, Facultad de Medicina, Universidad de la República. Uruguay.

2. Prof. Adjunto Clínica Ginecotológica A, Facultad de Medicina, Universidad de la República. Uruguay.

3. Prof. Adjunto Clínica Ginecotológica A, Facultad de Medicina, Universidad de la República. Uruguay.

Hospital Escuela del Litoral y Corporación Médica de Paysandú.

Correspondencia: Vanessa Guzzo. Wilson Ferreira Aldunate 1709, Paysandú, Uruguay.

Correo electrónico: vaneguzzo86@gmail.com.

Los autores declaran no tener conflicto de interés.

Aprobado post facto por el Comité de Etica en Investigacion del Sanatorio Americano.

Recibido: 22/9/20

Aprobado: 18/11/20

Attribution-NonCommercial 4.0 International (CC BY-NC 4.0)
} 


\section{Introducción}

La implantación del blastocisto fuera del revestimiento endometrial de la cavidad uterina se considera un embarazo ectópico ${ }^{(1)}$. Si bien es parte de las principales causas de muerte materna en el primer trimestre de embarazo, en los últimos años se viene presentando un fenómeno de disminución en las defunciones, hecho que podría explicarse por los avances en el diagnóstico precoz, consecuencia de un aumento de la sensibilidad en la determinación de la gonadotropina coriónica humana ( $\beta$-HCG) y la ecografía transvaginal de alta resolución realizada de forma tempra$n a^{(2,3)}$. La detección oportuna permite la generación de opciones terapéuticas diferentes al tratamiento quirúrgico, que van desde el manejo expectante al tratamiento médico. Es así que el tratamiento médico con metotrexate aparece como una alternativa eficaz y segura para las pacientes estables y con criterios específicos de selección ${ }^{(4)}$. Se estima que entre $60 \%-65 \%$ de los embarazos ectópicos podrán ser candidatos al tratamiento ${ }^{(3,5)}$. Aumentando, de este modo, la morbimortalidad materna y la conservación de la capacidad reproductiva de las mujeres que cursan un embarazo ectópico ${ }^{(1)}$.

En el contexto actual de la pandemia por Covid-19 algunas sociedades científicas consideran que el tratamiento médico con metotrexate es de primera línea y cobra mayor importancia para evitar el ingreso hospitalario y el abordaje quirúrgico ${ }^{(6)}$.

El objetivo del presente trabajo es reportar la experiencia del tratamiento médico con metotrexate en el departamento de Paysandú. Secundariamente, determinaremos la satisfacción de las usuarias frente a éste y su fertilidad posterior.

\section{Marco teórico}

El embarazo extrauterino o ectópico (EE), del griego ektopos (fuera de lugar), corresponde a la implantación del trofoblasto fuera del útero, comúnmente en la trompa y rara vez en el ovario o cavidad peritoneal ${ }^{(7)}$.

La incidencia del EE ha aumentado en las últimas décadas hasta alcanzar el 1\%-2\%, debido a tres condiciones: el incremento de los factores de riesgo, el incremento en la aplicación de técnicas de reproducción asistida y el uso de métodos diagnósticos más sensibles y específicos que permiten detectar casos asintomáticos ${ }^{(8)}$.

Sin embargo, la mortalidad materna ha disminuido gracias al diagnóstico precoz, determinado por la ecografía transvaginal, y determinaciones seriadas de $\beta$-HCG con una sensibilidad de $96 \%$, una especificidad de $97 \%$ y un valor predictivo positivo de $95 \%$, siendo las pruebas más eficientes para este diagnóstico ${ }^{(8)}$.

La detección precoz nos ha hecho evidente el curso natural de la enfermedad, con muchas gestaciones tuba- rias prácticamente indolentes y con una baja probabilidad de hemorragia repentina. Por lo cual, el tratamiento pasa a depender del lugar, del tamaño, del estado de la trompa y de los deseos de la paciente por conservar la fertilidad $^{(9)}$.

En el siglo XIX el ginecólogo británico Robert Lawson Tait impuso el concepto de que la extirpación del órgano enfermo era la única oportunidad que tenía la paciente para salvar su vida ${ }^{(10)}$. El abordaje en la actualidad tiene claramente dos vertientes: la quirúrgica laparoscópica, hasta ahora la más extendida, y la no quirúrgica con conducta expectante o tratamiento médico con metotrexate $^{(3)}$.

Algunos embarazos tubáricos serán reabsorbidos espontáneamente determinando la opción de conducta expectante, pero en un 10\%-30\% de ellos se requerirá un tratamiento quirúrgico posterior ${ }^{(9)}$.

El metotrexate es un antagonista del ácido fólico que se une al sitio catalítico de la dihidrofolatoreductasa (DHFR), enzima que reduce el ácido fólico a tetrahidrofolato. Esta inhibición interrumpe la síntesis y reparación del ADN y las replicaciones celulares, inactivando de ese modo la proliferación del trofoblasto ${ }^{(3,11,12)}$. Está indicado en pacientes hemodinámicamente estables, con $\beta$-HCG $<5.000$, un diámetro de masa $<4 \mathrm{~cm}$ y en ausencia de latido embrionario ${ }^{(13,14)}$. Su uso se contraindica ante discrasia sanguínea preexistente, transaminasas elevadas o hepatopatía crónica por su toxicidad para los hepatocitos, elevación de creatinina $\geq 1,5 \mathrm{mg} / \mathrm{dl}$, dado que su excreción es renal, úlcera péptica activa, lactancia materna, dudas ante embarazo intrauterino, inmunodeficiencia y enfermedad pulmonar activa, excepto asma ${ }^{(13-16)}$.

Existen diferentes técnicas de aplicación del metotrexate: vía sistémica o vía local mediante inyección directa. La vía sistémica es más práctica, sencilla de administrar, no invasiva, y no requiere destreza quirúrgi$\mathrm{ca}^{(9,17)}$. El esquema de dosis utilizado es debatido. Hay tres protocolos publicados para su administración sistémica: dosis única, dos dosis y dosis múltiple ${ }^{(16,18)}$. El régimen de dos dosis puede considerarse como una alternativa al régimen de dosis única en mujeres con un alto valor inicial de $\beta$-HCG ${ }^{(13)}$.

Con dosis única, la tasa de éxito a nivel internacional ronda el 70\%-88\%, es tan efectivo como la dosis múltiple, con menor costo y menores efectos adversos ${ }^{(16,19-21)}$.

En el análisis de costos la diferencia a favor del tratamiento médico versus resolución quirúrgica es destacable, por no necesitar hospitalización ni ingreso a block $^{(10)}$.

La tasa de efectos adversos es por debajo de $10 \%$, generalmente son dependientes de la dosis y duración del tratamiento. Corresponden a úlcera péptica, estoma- 
titis, gastritis, náuseas y vómitos, dolor abdominal a los 2-3 días del inicio, posiblemente por el efecto citotóxico en el tejido trofoblástico, flatulencias, mielotoxicidad, hepatotoxicidad y fotosensibilidad, que se puede disminuir con aumento de la ingesta de líquidos y evitando la exposición solar. Los menos frecuentes son neumonitis $\mathrm{y}$ alopecia que revierte una vez culminado el tratamiento ${ }^{(12-14,16,21)}$.

Una vez seleccionadas las pacientes y previo al inicio de la terapéutica se debe informar con claridad las ventajas y desventajas de cada uno de los tratamientos, debiendo dar la paciente su consentimiento por escrito. Es importante educar a las usuarias sobre los síntomas de ruptura tubárica y enfatizar en la necesidad de buscar atención médica inmediata si éstos ocurren. La búsqueda de una nueva gestación se deberá relegar hasta pasado los tres meses de la inyección de metotrexate por su posible efecto teratogénico ${ }^{(9,14)}$. La monitorización intensiva de $\beta$-HCG al cuarto y séptimo día en suero es obligatoria para detectar un fracaso inminente del tratamiento, por lo que el seguimiento será clínico y paraclínico, existiendo indicación de ecografía solo en caso de sospecha de rotura tubárica reciente, la cual ronda en un $7 \%{ }^{(16,17)}$. Si el valor de $\beta$-HCG entre los días 4-7 disminuye un 15\% o menos, se debe la repetir dosis de metotrexate; si ocurre lo contrario, se realizará $\beta$-HCG semanalmente hasta obtener niveles inferiores a $15 \mathrm{IU} / \mathrm{l}^{(11,13,16)}$.

Hablamos de persistencia trofoblástica cuando los niveles de $\beta$-HCG ascienden o no caen lo esperado, correspondiendo a un $8 \%-10 \%$ de los tratamientos médicos, $8 \%$ de las salpingostomías laparoscópicas y $4 \%$ de las laparotómicas ${ }^{(22)}$.

El riesgo de recurrencia ronda 5\%-25\% según diferentes estudios y no varía con la modalidad del tratamiento instaurado ${ }^{(23)}$.

Una vez superado el cuadro, se estima una fertilidad posterior, según Bonin y colaboradores, de 75\%-80,7\%, siendo similar para las diferentes opciones de tratamiento ${ }^{(10,13,24)}$.

\section{Metodología}

Realizamos un estudio retrospectivo, observacional, de una serie de casos clínicos con EE tubario manejados con tratamiento médico en el servicio de Ginecología y Obstetricia de Corporación Médica de Paysandú (COMEPA) y del Hospital Escuela del Litoral (HEL).

Se recogieron los datos del sistema de información quirúrgica de ambos centros, donde constaba el diagnóstico de EE para nuestro $n$ total de la muestra y el registro de las oficinas de farmacia de ambos centros sobre el uso de metotrexate en el servicio de ginecología durante el período $1^{\circ}$ de enero de 2014 al 31 de diciembre de 2017, comprendiendo cuatro años.
El departamento de Paysandú tiene una población de 113.107 habitantes, 23.439 son mujeres en edad reproductiva. Dentro de la localidad hay dos centros que cuentan con block quirúrgico que fueron incluidos en nuestro trabajo (COMEPA y HEL), por lo que estimamos el haber accedido a la mayoría de los cuadros de EE en mujeres del departamento.

El 10 de octubre 2018 se llamó telefónicamente a todas las pacientes en las que se realizó tratamiento médico con metotrexate a dosis de $50 \mathrm{mg} / \mathrm{m}^{2}$ intramuscular, se efectuó un interrogatorio basado en su deseo gestacional, embarazo espontáneo posterior al tratamiento y su experiencia con el uso de metotrexate (anexo 1).

Se incluyeron las pacientes con diagnóstico de EE que recibieron metotrexate a dosis de $50 \mathrm{mg} / \mathrm{m}^{2}$ intramuscular y se excluyeron en las que se realizó tratamiento quirúrgico o conducta expectante.

Como variables utilizamos: prestador de salud, edad de las pacientes, factores de riesgo (antecedentes de abortos espontáneos, dispositivos intrauterinos, antecedente de EE previo, infertilidad), método anticonceptivo utilizado previamente, edad gestacional al momento del diagnóstico, diámetro de la masa anexial, nivel de $\beta$-HCG día 1, día 4 y día 7, tiempo transcurrido desde el inicio del tratamiento hasta la negativización de la $\beta$-HCG, tipo de resolución del cuadro clínico, complicaciones, fertilidad posterior de manera espontánea, tiempo transcurrido desde la $\beta$-HCG hasta concepción y conformidad con el tratamiento.

Se consideró fracaso el tratamiento médico cuando fue necesario tratamiento quirúrgico, ya sea por sospecha clínica de rotura, o si luego de la segunda dosis de 50 $\mathrm{mg} / \mathrm{m}^{2}$ de metotrexate intramuscular, no se logró un descenso de al menos $15 \%$ de $\beta$-HCG entre el cuarto y séptimo día.

La fertilidad futura se definió como el tiempo que transcurrió hasta la ocurrencia de una gestación intrauterina viable espontánea.

Para el procesamiento de datos se utilizó el sistema IBM SPSS Statistics 24.0 (Statistical Package for the Social Sciences).

Se presenta el artículo posfacto al Comité de Ética en la Investigación de Sanatorio Americano (CEI- SASA). Se tomaron medidas para garantizar la confidencialidad de datos de acuerdo a la declaración de Helsinki y se realizó consentimiento informado a todas las participantes.

\section{Resultados}

En los cuatro años de estudio hubo $67 \mathrm{EE}$, de ellos, 13 recibieron tratamiento médico, $77 \%$ en la salud privada y el $23 \%$ restante en la salud pública.

De las características de la población en las que se realizó metotrexate (tabla 1), los rangos de edades fue- 
Tabla 1. Características de las mujeres con embarazo ectópico tubario tratado con metotrexate.

\begin{tabular}{lc}
\hline Edad & $29,6 \pm 5,78$ \\
Edad gestacional en semanas & $6,2 \pm 1,2$ \\
Factores de riesgo para embarazo ectópico & $6(46,1 \%)$ \\
Antecedente de embarazo ectópico & $4(30,7 \%)$ \\
Diámetro de masa anexial en mm & $27 \pm 8,4$ \\
1er $\beta$-HCG en $\mathrm{mUl} / \mathrm{ml}$ & $11.833 \pm 29.851,7$ \\
4ta $\beta$-HCG en $\mathrm{mUl} / \mathrm{ml}$ & $5.537 \pm 5.890,6$ \\
7ta $\beta$-HCG en mUl/ml & $5.003 \pm 7.270,4$ \\
Tiempo de negativización $\beta$-HCG en días & $32 \pm 14,5$ \\
Embarazo espontáneo & $3(25 \%)$ \\
Conformidad con el tratamiento & $11(84 \%)$ \\
\hline
\end{tabular}

ron de 21 a 40 años, con una media de 29,6 años y un desvío estándar de 5,78 años. Seis pacientes presentaban factores de riesgo, de las cuales cuatro tenían antecedente de EE previo. Sobre la anticoncepción utilizada previamente, seis pacientes no utilizaban ningún método, cuatro usaban anticoncepción hormonal combinada, una de barrera y dos usuarias sin datos. La edad gestacional de presentación fue entre 4 y 8 semanas con una media de 6,2 semanas. En cuanto al diámetro de la masa anexial se obtuvo una media de $27 \mathrm{~mm}$ con un desvío estándar de $8,7 \mathrm{~mm}$, para un total de 11 pacientes. Los valores iniciales de $\beta$-HCG presentaron una media de 11833 y un desvío estándar de 29851,7. El nivel al cuarto día presentó una media de 5537 para el total de pacientes y un desvío estándar de 5890,6. El número de $\beta$-HCG realizadas al séptimo día fueron 12 de las 13 , con una media de 5003 y un desvío estándar de 7.270,4.

En cuanto al tiempo transcurrido desde la primera $\beta$-HCG hasta tornarse negativa varió entre 7 a 57 días, con un promedio de 32 días para un total de siete pacientes. Hay dos pacientes, con dificultad en el seguimiento, que se consideraron dentro del éxito en el tratamiento por no registrarse actos quirúrgicos en ellas. Las otras cuatro restantes culminaron con tratamiento quirúrgico por complicaciones o falla en el tratamiento.

Hoy día, para la indicación de metotrexate, se requiere de hemodinámica estable, con $\beta$-HCG $<5000$, un diámetro de masa $<4 \mathrm{~cm}$ y en ausencia de latido embrionario. Los médicos tratantes de cuatro mujeres no siguieron dicha recomendación, como se visualiza en la tabla 2. De igual modo, se obtuvo un éxito en el tratamiento con metotrexate sistémico a dosis de $50 \mathrm{mg} / \mathrm{m}^{2}$ intramuscular en nueve pacientes de un total de 13 , correspondiendo a $69,2 \%$.

Según el cuestionario de conformidad con el tratamiento planteado, de las 13 pacientes solo dos lo indicaron como una experiencia negativa, perteneciendo a los ectópicos rotos; se obtuvo 84\% de aceptación. La fertilidad futura ocurrió en tres de las 11 pacientes con deseo concepcional (25\%). El tiempo que transcurrió para la nueva gestación fue entre 16 meses y 35 meses con una media de 23 meses.

\section{Discusión y conclusiones}

El éxito global del tratamiento con metotrexate sistémico para el EE, definido como la resolución de éste sin necesidad de cirugía, oscila entre $75 \%$ a $95 \%$, según la bibliografía internacional ${ }^{(4,12,25)}$. En nuestro departamento, en el año 2001, Leoni y colaboradores reportaron una serie de cinco EE tratados con metotrexate con un porcentaje de éxito de $100 \%{ }^{(26)}$. Los valores obtenidos en nuestro caso rondaron el $69,2 \%$ de éxito, por debajo de lo que informan datos nacionales e internacionales.

Esta diferencia con respecto a la literatura se puede atribuir a la disparidad entre los médicos tratantes para la selección de las pacientes candidatas a metotrexate, si bien ninguna presentaba contraindicación absoluta para su realización, no todas cumplían con los criterios para su uso ${ }^{(15)}$. En la tabla 2, en negrita, destacamos a las cuatro pacientes que quedarían por fuera si aplicáramos el protocolo RCOG (Royal College of Obstetricians and Gynaecologists) $)^{(15)}$, aunque se puede observar que dos de ellas evolucionaron favorablemente al tratamiento. Si excluyéramos a las cuatro pacientes que no cumplían con criterios clínicos y paraclínicos, nuestro porcentaje de éxito para tratamiento médico ascendería a 77,7\%.

Cabe destacar que dos usuarias con tratamiento exitoso ya habían recibido, en 2013 y 2014, metotrexate a la misma dosis y una de ellas logró actualmente un embarazo espontáneo sin complicaciones, por lo que el $100 \%$ de las pacientes que habían tenido un EE previamente tratado con metotrexate, volvieron a tener éxito. Incluso una de las pacientes que respondió nuevamente a metotrexate, tenía un valor de $\beta$-HCG extremadamente elevado $(115000 \mathrm{mUI} / \mathrm{ml})$, siendo catalogado como predictor de falla ${ }^{(15)}$.

Por otra parte, hubo 67 EE en cuatro años, se realizó tratamiento quirúrgico a 51 pacientes $(76 \%)$, metotrexate a $13(19 \%)$ y se planteó conducta expectante en tres pacientes $(5 \%)$, datos similares a la revisión de Hsu, JY ${ }^{(27)}$.

De las 51 pacientes intervenidas quirúrgicamente, $69 \%$ correspondía al ámbito público, donde se lleva a cabo formación universitaria; estos números son discordantes con los obtenidos por la revisión de Hsu, JY, don- 


\begin{tabular}{|c|c|c|c|c|c|c|c|}
\hline $\begin{array}{c}E G \\
\text { (semanas) }\end{array}$ & $1 d \beta-H C G$ & $4 d \beta-H C G$ & $7 d \beta-H C G$ & ECO & TPU mm & Resolución & Fertilidad \\
\hline Sin dato & 2.463 & 1.523 & 289 & EE der + líq escaso & $25 \times 35$ & $\begin{array}{c}\text { A los } 30 \text { dias } \beta \text {-HCG } \\
\text { negativa }\end{array}$ & Sí 17 meses \\
\hline 5 & 3.796 & 2.877 & 1.096 & $\begin{array}{l}\text { Anexos normal, líq } \\
\text { escaso }\end{array}$ & & $\begin{array}{l}\text { Laparotomía } \\
\text { hemoperitoneo } \\
\text { izquierdo }\end{array}$ & No aún \\
\hline Sin dato & 4.853 & 4.902 & Sin dato & SG izquierdo & Sin dato & $\begin{array}{c}\text { Sin dato de } \beta-H C G \\
\text { controles }\end{array}$ & Sí 35 meses \\
\hline 7 & 7.560 & 6.260 & 1.958 & Imagen de EE & $18 \times 24$ & $\begin{array}{l}\text { Sin dato de } \beta-H C G \\
\text { controles }\end{array}$ & No aún \\
\hline 6 & 2.762 & 18.501 & 23.843 ( $2^{\mathrm{a}}$ dosis $)$ & SG con latido & $30 \times 40$ & $\begin{array}{l}\text { Salpingectomía } \\
\text { izquierda por falla del } \\
\text { tratamiento }\end{array}$ & No aún \\
\hline 7 & 3.828 & 4.169 & 2.393 & Imagen anecoica der & $32 \times 17$ & $\begin{array}{c}\text { A los } 57 \text { días } \beta \text {-HCG } \\
\text { negativa }\end{array}$ & No aún \\
\hline Sin dato & 28 & 11 & 0 & $\begin{array}{l}\text { Imagen a derecha } \\
\text { vascularizada }\end{array}$ & $32 \times 18$ & $\begin{array}{c}\text { A los } 7 \text { días } \beta-H C G \\
\text { negativa }\end{array}$ & $\begin{array}{l}\text { Sin deseo } \\
\text { gestacional }\end{array}$ \\
\hline 4 & 738 & 440 & 332 & Imagen de EE & $27 \times 27$ & $\begin{array}{c}\text { A los } 26 \text { días } \beta \text {-HCG } \\
\text { negativa }\end{array}$ & No aún \\
\hline 6 & 5.800 & 10.420 & 8.460 & $\begin{array}{l}\text { Imagen de EE } \\
\text { derecho }\end{array}$ & $13 \times 16$ & $\begin{array}{c}\text { Laparotomía } \\
\text { hemoperitoneo }\end{array}$ & No aún \\
\hline 5 & 1.501 & 843 & 653 & $\begin{array}{l}\text { Imagen de EE } \\
\text { izquierdo }\end{array}$ & $25 \times 28$ & $\begin{array}{c}\text { A los } 39 \text { días } \beta \text {-HCG } \\
\text { negativa }\end{array}$ & No aún \\
\hline 7 & 4.344 & 4.170 & $3.990\left(2^{\mathrm{a}}\right.$ dosis MTX) & $\begin{array}{l}\text { Imagen de EE } \\
\text { derecho }\end{array}$ & $15 \times 17$ & $\begin{array}{l}\text { Laparoscopía por } \\
\text { falla tratamiento }\end{array}$ & Sí 16 meses \\
\hline 8 & 1.165 & 878 & 689 & $\begin{array}{l}\text { Imagen anecoica } \\
\text { der, Doppler neg }\end{array}$ & $32 \times 27$ & $\begin{array}{c}\text { A los } 24 \text { dias } \beta \text {-HCG } \\
\text { negativa }\end{array}$ & No aún \\
\hline 7 & 115.000 & 16.988 & 16.336 & $\begin{array}{l}\text { SG derecho } \\
\text { con latido }\end{array}$ & $14 \times 14$ & $\begin{array}{c}\text { A los } 41 \text { días } \beta \text {-HCG } \\
\text { negativa }\end{array}$ & No aún \\
\hline
\end{tabular}

de en los centros docentes había 16\% más de probabilidades de recibir metotrexate ${ }^{(27)}$. Es necesario mencionar que en nuestro departamento no se usa metotrexate de forma ambulatoria, y que todas las pacientes tienen como prestador de salud COMEPA o HEL, por lo cual contamos con un bajo sesgo en el número de pacientes con tratamiento médico.

Cuando analizamos los factores de riesgo y las características de la población, la edad tuvo un rango de 21 a 40 años, con una media de 29,6 años y un desvío estándar de 5,78 años, lo que concuerda con la revisión de Hsu JY y colaboradores, en julio de $2017^{(27)}$.

Tenían factores de riesgo para embarazo extrauterino $6(46,1 \%)$ de las 13 pacientes, porcentaje conocido internacionalmente ${ }^{(15)}$. Sobre la anticoncepción utilizada previamente, seis pacientes no utilizaban ningún mé- todo, a diferencia con lo publicado por Furlong en 2002, que aseguraba que el uso de anticonceptivos orales de progestágeno, implantes de progestágeno, dispositivos intrauterinos y ligaduras de trompas, era lo que predisponía a esta patología en la población general ${ }^{(28)}$.

Otra variable que se desprende de nuestro estudio es el tiempo transcurrido hasta la negativización del valor de $\beta$-HCG, que varió entre 7 a 57 días, rondando los 32 días. Tiempo similar es reportado en la bibliografía, donde se estima una resolución completa a las 2-4 semanas, con un máximo de ocho semanas ${ }^{(13)}$. Sí debemos resaltar que dos pacientes no continuaron con los controles de seguimiento correspondientes. Dai y colaboradores, en un artículo publicado recientemente, proponen eliminar la medición de $\beta-\mathrm{HCG}$ al cuarto día con el fin de disminuir costos y obtener una mejor adherencia al 
seguimiento ambulatorio, pudiendo ser una alternativa beneficiosa sobre todo en nuestro medio ${ }^{(29)}$.

Se presentaron cuatro complicaciones en las 13 pacientes, dos pacientes fueron intervenidas por vía laparotómica con diagnóstico presuntivo de rotura, dos culminaron con tratamiento quirúrgico laparoscópico por falla en el tratamiento médico.

Sobre la conformidad con el tratamiento planteado, solo dos de las 13 pacientes lo indicaron como una experiencia negativa, por la intensidad del dolor y el requerimiento de varios días de internación. El 84\% lo consideró aceptable, destacando a su vez que aquellas pacientes que lo recibieron previamente, lo volvieron a solicitar en esta nueva gestación ectópica. Ninguna relató como experiencia desfavorable la presencia de efectos adversos. La publicación, en 2001, de Sowter y colaboradores, que compara resultados psicológicos y físicos en mujeres con tratamiento médico y cirugía laparoscópica, mostró puntaciones a favor del metotrexate en cuanto al funcionamiento físico, sin diferencias significativas en las puntaciones de estado de ansiedad y depresión ${ }^{(30)}$.

Con respecto a la fertilidad futura, de las mujeres que tuvieron éxito en el tratamiento solo el $25 \%$ logró un embarazo viable espontáneo, aunque una paciente no tenía deseo concepcional en ese momento. Valor por debajo de la bibliografía internacional, que oscila entre $67 \%$ $80,7 \%{ }^{(24,32)}$. El tiempo transcurrido hasta la concepción fue muy variable, de 16 a 35 meses $^{(10)}$.

Si bien una limitante de nuestro trabajo es el hecho de ser retrospectivo, pudiéndose ver afectado por sesgos innatos a este tipo de estudio, sumado al número pequeño de la muestra, su realización nos permitió reflejar el análisis de nuestra experiencia en esta práctica clínica, dar a conocer las diferentes opciones que se tienen hoy día para el manejo de la patología y objetivar la variabilidad de técnicas aplicadas según el profesional actuante, por lo que sería conveniente realizar a nivel nacional un estudio prospectivo, multicéntrico, con un protocolo determinado para conocer la tasa de éxito nacional y comenzar a instaurar en nuestro país este tratamiento beneficioso.

Los autores concluyen que el uso de metotrexate en el EE se practica en Paysandú desde hace casi una década con buenos resultados y una buena aceptación por parte de las usuarias.

\section{Agradecimientos}

A los Dres. Ricardo Medina y Marcos López, ambos jefes de cada servicio participante. A los Dres. Leoni, Rezzano, Pintos, García, Laluz y a la Dra. Lijstenstein por facilitarnos el acceso a su artículo.

\section{Summary}

Introduction: In recent years there has been a decrease in deaths related to ectopic pregnancy, thanks to progress made in early diagnosis, which in turn allowed for medical treatment with methotrexate in selected patients.

The study's first objective is to report the experience of methotrexate treatment in the Department of Paysandú and its secondary objective is to determine users' satisfaction towards this treatment and find about their subsequent fertility.

Method: a retrospective, observational study is presented of tubal ectopic pregnancies treated with methotrexate in Paysandú, from January 1, 2014 to December 31, 2017, during a 4-year period. Failure of medical treatment was defined as the cases requiring surgical treatment and subsequent fertility was defined as the time it took to achieve a spontaneous intrauterine gestation.

Results: 67 ectopic pregnancies were recorded in four years, 13 of which received intramuscular methotrexate with a success rate of $69.2 \%$. Subsequent fertility was found to be $25 \%$.

Conclusions: methotrexate has been used to treat ectopic pregnancies in Paysandú for almost a decade, with good results and acceptable rates of user satisfaction.

\section{Resumo:}

Introdução: nos últimos anos, observou-se uma diminuição dos óbitos relacionados à gravidez ectópica, graças aos avanços no diagnóstico precoce, que por sua vez abriram as portas para o tratamento médico com metotrexato em pacientes selecionadas.

Objetivo: o objetivo deste trabalho é relatar a experiência do tratamento com metotrexato no Departamento de Paysandú e determinar secundariamente a satisfação das usuárias com o mesmo e sua consequente fertilidade.

Métodos: apresenta-se um estudo retrospectivo e observacional de gestações ectópicas tubárias tratadas com metotrexato em Paysandú, durante o período de $1^{\circ}$ de janeiro de 2014 a 31 de dezembro de 2017. Considerou-se como falha do tratamento médico quando o tratamento cirúrgico foi necessário e definiu-se fertilidade futura como o tempo decorrido até a obtenção de uma gravidez intrauterina espontânea.

Resultados: foram registradas 67 gestações ectópicas em quatro anos, 13 receberam metotrexato intramuscular com taxa de sucesso de $69,2 \%$. Uma fertilidade subsequente de $25 \%$ foi determinada. 
Conclusões: o uso do metotrexato na gravidez ectópica é praticado em Paysandú há quase uma década com bons resultados e boa aceitação pelas usuárias.

\section{Bibliografía}

1. Embarazo ectópico. En: Cunningham F, Leveno K, Bloom S, Hauth J, Rouse D, Spong C. Williams obstetricia. 23 ed. México: Mc Graw-Hill Interamericana, 2011:238-55.

2. Centers for Disease Control and Prevention. Ectopic pregnancy mortality - Florida, 2009-2010. MMWR Morb Mortal Wkly Rep 2012; 61(6):106-9. Disponible en: https://www.cdc.gov/mmwr/preview/mmwrhtml/mm6106a2.htm. [Consulta: 17/6/20].

3. Salvador Alarcón C, Callejo Olmos J, Laílla Vicens J. Embarazo ectópico. En: González Merlo J, Laílla Vicens J, Fabre González E, González Bosquet E. Obstetricia. 6 ed. Barcelona: Elsevier, 2013:331-45.

4. Mol F, Mol B, Ankum W, van der Veen F, Hajenius P. Current evidence on surgery, systemic methotrexate and expectant management in the treatment of tubal ectopic pregnancy: a systematic review and meta-analysis. Hum Reprod Update 2008; 14(4):309-19. doi: 10.1093/humupd/dmn012

5. Embarazo ectópico. En: Fescina R, De Mucio B, Ortiz E, Jarquin D. Guías para la atención de las principales emergencias obstétricas. Publicación científica CLAP/SMR 1594. Montevideo: CLAP/SMR, 2012:25-32. Disponible en: http://www.paho.org/clap/index.php?option=com_docman\&view=download\&category_slug=salud-de-mujer-reproductiva-materna-y-perina-

tal\&alias=279-guias-para-la-atencion-de-las-principales-emergencias-obstetricas-1\&Itemid=219\&lang=es. [Consulta: $17 / 6 / 20]$.

6. Mallick R, Odejinmi F, Clark T. Covid 19 pandemic and gynaecological laparoscopic surgery: knowns and unknowns. Facts Views Vis Obgyn 2020; 12(1):1-5. Disponible en: https://mk0britishsociep8d9m.kinstacdn.com/wp-content/uploads/2020/04/Covid-19-pandemic-and-gynaecological-laparoscopic-surgery-knowns-and-unknowns.pdf [Consulta: $17 / 6 / 20]$.

7. Ramanah R, Marguier I, Mottet N, Magnin C, RiethmuIler D. Embarazo extrauterino. EMC Ginecol Obstetr 2018; 54(3):1-14. doi: 10.1016/S1283-081X(18)91440-2

8. Sociedad Española de Ginecología y Obstetricia. Embarazo ectópico. Prog Obstet Ginecol 2007; 50(6):377-85. Disponible en: http://www.elsevier.es/es-revista-progresos-obstetricia-ginecologia-151-pdf-13106416-S300. [Consulta: enero 2017].

9. Casas M, Sánchez J. Consideraciones éticas sobre el uso de metotrexate en el embarazo tubario (ET). Acta Bioeth 2012; 18(2):147-53. Disponible en: https://scielo.conicyt.cl/scielo.php?script=sci_arttext\&pid=S1726-569X2012000200002. [Consulta: 17/6/20].

10. Ruiz-Velasco V. Manejo actual del embarazo ectópico en la paciente estéril y en la que se desea conservar su fertilidad fu- tura: actualización, Prog Obstet Ginecol 2010; 53(9):351-63. doi:10.1016/j.pog.2010.04.001

11. Song T, Kim M, Kim M, Jung Y, Yun B, Seong S. Single-dose versus two-dose administration of methotrexate for the treatment of ectopic pregnancy: a randomized controlled trial. Hum Reprod 2016; 31(2):332-8. doi: 10.1093/hum$\mathrm{rep} / \operatorname{dev} 312$

12. Practice Committee of American Society for Reproductive Medicine. Medical treatment of ectopic pregnancy: a committee opinion. Fertil Steril 2013; 100(3):638-44. doi: 10.1016/j.fertnstert.2013.06.013

13. Sánchez-Hidalgo L, López de la Manzanara C, Alpuente A, Garrido R, Sánchez- Hipólito L, González-López A. Evaluación del tratamiento médico del embarazo ectópico con metotrexato, Clin Invest Gin Obst 2012; 39(6):249-52. Disponible en: https://vdocuments.site/evaluacion-del-tratamiento-medico-del-embarazo-ectopico-con-metotrexato.html. [Consulta: 17/6/20].

14. Centre de Medicina Fetal i Neonatal de Barcelona. Protocolo: gestación ectópica tubárica y no tubárica. Barcelona: Hospital Clínic, Universitat de Barcelona, 2018. Disponible en: https://medicinafetalbarcelona.org/protocolos/es/patologia-materna-obstetrica/gestaci $\% \mathrm{C} 3 \% \mathrm{~B} 3 \mathrm{n} \% 20$ ect $\% \mathrm{C} 3 \% \mathrm{~B} 3$ pica.pdf. [Consulta: 20 de dic. de 2020]

15. Royal College of Obstetricians \& Gynaecologists. Diagnosis and management of ectopic pregnancy: Green-top Guideline No. 21. BJOG 2016; 123(13):e15-e55. doi: $10.1111 / 1471-0528.14189$

16. American College of Obstetricians and Gynecologists' Committee on Practice Bulletins-Gynecology. ACOG Practice Bulletin No. 193: tubal ectopic pregnancy. Obstet Gynecol 2018; 131(3):e91-e103. doi: 10.1097/AOG. 0000000000002560

17. Kim J, Jung Y, Lee D, Jee B. Pretreatment serum human chorionic gonadotropin cutoff value for medical treatment success with single-dose and multi-dose regimen of methotrexate in tubal ectopic pregnancy. Obstet Gynecol Sci 2017; 60(1):79-86. doi: 10.5468/ogs.2017.60.1.79

18. Marret H, Fauconnier A, Dubernard G, Misme H, Lagarce L, Lesavre M, et al. Overview and guidelines of off-label use of methotrexate in ectopic pregnancy: report by CNGOF. Eur J Obstet Gynecol Reprod Biol 2016; 205:105-9. doi: 10.1016/j.ejogrb.2016.07.489

19. Yang C, Cai J, Geng Y, Gao Y. Multiple-dose and double-dose versus single-dose administration of methotrexate for the treatment of ectopic pregnancy: a systematic review and meta-analysis. Reprod Biomed Online 2017; 34(4):383-91. doi: 10.1016/j.rbmo.2017.01.004

20. Guvendag Guven E, Dilbaz S, Dilbaz B, Aykan Yildirim B, Akdag D, Haberal A. Comparison of single and multiple dose methotrexate therapy for unruptured tubal ectopic pregnancy: a prospective randomized study. Acta Obstet Gynecol Scand 2010; 89(7):889-95. doi: 10.3109/00016349. 2010.486825 
21. Farquhar C. Ectopic pregnancy. Lancet 2005; 366(9485):583-91. doi: 10.1016/S0140-6736(05)67103-6

22. Graczykowski J, Mishell DJr. Methotrexate prophylaxis for persistent ectopic pregnancy after conservative treatment by salpingostomy. Obstet Gynecol 1997; 89(1):118-22. doi: 10.1016/s0029-7844(96)00370-5

23. Panelli D, Phillips C, Brady P. Incidence, diagnosis and management of tubal and nontubal ectopic pregnancies: a review. Fertil Res Pract 2015; 1:15. doi: 10.1186/s40738015-0008-z

24. Bonin L, Pedreiro C, Moret S, Chene G, Gaucherand P, Lamblin G. Predictive factors for the methotrexate treatment outcome in ectopic pregnancy: A comparative study of 400 cases. Eur J Obstet Gynecol Reprod Biol 2017; 208:23-30. doi: 10.1016/j.ejogrb.2016.11.016

25. Bueno F, Sáez J, Ocaranza M, Conejeros C, Vaccarezza I, Borquez $\mathbf{P}$, et al. Tratamiento médico del embarazo ectópico. Rev Chil Obstet Ginecol 2002; 67(3):173-79. doi: $10.4067 / \mathrm{s} 0717-75262002000300001$

26. Leoni C, García F, López M, Pintos S, Rezzano R, Lijtenstein M, et al. Tratamiento médico del embarazo ectópico no complicado. Experiencia con el empleo de metotrexate. En: Sociedad Ginecotológica del Uruguay. XIII Congreso
Uruguayo de Ginecotología; 2001 mayo 10-12; Montevideo, Uruguay.

27. Hsu J, Chen L, Gumer A, Tergas A, Hou J, Burke W, et al. Disparities in the management of ectopic pregnancy. Am J Obstet Gynecol 2017; 217(1):49.e1-49.e10. doi: 10.1016/ j.ajog.2017.03.001

28. Furlong L. Ectopic pregnancy risk when contraception fails. A review. J Reprod Med 2002; 47(11):881-5.

29. Dai Y, Zhang G, Zhu L, Lang J, Liu Z. Routine $\beta$-Human Chorionic Gonadotropin Monitoring for Single-Dose Methotrexate Treatment in Ectopic Pregnancy. J Minim Invasive Gynecol 2017; 24(7):1195-9. doi: 10.1016/j.jmig.2017. 07. 025

30. Sowter M, Farquhar C, Petrie K, Gudex G. A randomised trial comparing single dose systemic methotrexate and laparoscopic surgery for the treatment of unruptured tubal pregnancy. BJOG 2001; 108(2):192-203. doi: 10.1111 /j.14710528.2001.00038.x

31. Fernández H, Capmas P, Lucot J, Resch B, Panel P, Bouyer J. Fertility after ectopic pregnancy: the DEMETER randomized trial. Hum Reprod 2013; 28(5):1247-53. doi: 10.1093/humrep/det037

\section{Contribución de autores}

Vanessa Guzzo, https://orcid.org/0000-0002-8578-4523. Concepción, diseño, análisis, interpretación de resultados y redacción. Sebastián Ben, https://orcid.org/0000-0001-7664-4687. Revisor crítico, interpretación de resultados y redacción.

Natalia Sica, https://orcid.org/0000-0003-3549-8972. Análisis y revisión crítica. 
Anexo 1. Interrogatorio telefónico.

1. ¿Considera usted su experiencia en el tratamiento con metotrexate como positiva o negativa?

2. ¿Luego de su tratamiento, usted ha tenido deseo concepcional?

3. ¿En caso de ser positiva la respuesta anterior, ha logrado un embarazo espontáneo? 